\title{
Aceptación y uso de los sistemas e-learning por estudiantes de grado de ecuador: El caso de una universidad estatal
}

\author{
Richard Ramirez-Anormaliza ${ }^{1}$ (D), Ferrán Sabaté2 (iD), Xavier Llinàs-Audet²(iD), Oriol Lordan² (iD \\ ${ }^{1}$ Universidad Estatal de Milagro, Instituto Superior Tecnológico Bolivariano de Tecnología (Ecuador) \\ ${ }^{2}$ Universitat Politècnica de Catalunya (Spain) \\ rramireza@unemi.edu.ec,.ferran.sabate@upc.edu, xavier.llinas@upc.edu, oriol.lordan@upc.edu
}

Received June, 2016

Accepted November, 2016

\section{Resumen}

Objeto: El objetivo de esta investigación fue adaptar el Modelo de Aceptación de la Tecnología (TAM) para predecir el uso y la intención de uso de los sistemas e-learning entre los estudiantes de grado de una universidad estatal en Ecuador, con la intención de mejorar el entendimiento de los factores que puedan potenciar el uso de estos sistemas y facilitar la aplicación de políticas para incrementar los beneficios que éstos aportan al proceso de enseñanza y aprendizaje.

Diseño/metodología/enfoque: El análisis tiene fundamento epistemológico en lo empíricoinductivo, basado en la observación de la percepción. En base a la revisión de la literatura científica sobre la evaluación y aceptación de los sistemas e-learning, se ha adaptado el modelo al caso de Ecuador y se ha elaborado un cuestionario con 52 ítems basado en escalas de Likert. El instrumento fue enviado por correo electrónico a 600 estudiantes de grado de una universidad estatal en Ecuador, respondiendo satisfactoriamente 423 de ellos. Se realizaron comprobaciones de validez y fiabilidad del modelo de ecuaciones estructurales por mínimos cuadrados parciales (PLS), con el soporte del software estadístico SmartPLS. 
Aportaciones y resultados: Los resultados indican que todas las hipótesis del TAM se cumplen en el contexto ecuatoriano, al momento de evaluar la aceptación de los sistemas elearning entre los estudiantes de grado de una universidad estatal. El principal aporte fue identificar que el entretenimiento percibido, la influencia social y la auto-eficacia computacional, tienen un efecto directo sobre los dos constructos principales del TAM, facilidad de uso percibida (PEOU) y utilidad percibida (PU). También se encontró que la satisfacción (S) recibe una elevada influencia directa de PU y a su vez $\mathrm{S}$ influye en el uso de los sistemas e-learning, siendo el primero un aporte específico de este estudio. El soporte técnico reflejó no tener influencia sobre los constructos fundamentales del TAM.

Limitaciones: La investigación fue realizada a los estudiantes de grado en una sola universidad estatal categoría B de Ecuador, siendo la realidad de este país más compleja, al existir cuatro categorías con características distintas. Tampoco se han considerado la edad, género, estudiantes de posgrado, condición socio económica, entre otras características de los estudiantes que puede afectar la investigación.

Implicaciones prácticas: La identificación de los factores que influyen en la aceptación y uso de los sistemas e-learning, contribuirá a crear y mejorar los entornos de enseñanza aprendizaje para los estudiantes universitarios. Permitiendo explotar con mayor eficiencia los beneficios de estas herramientas tecnológicas, adaptándolas a las políticas de gestión de las instituciones de educación superior.

Originalidad / Valor añadido: Este artículo presenta datos empíricos sobre el uso y aceptación de los sistemas e-learning en los estudiantes de una determinada universidad de Ecuador. No se evidencian estudios similares en el país y el modelo puede ser considerado para futuros estudios de alcance nacional.

Palabras clave: e-learning, TAM, PLS, SEM, Modelo de ecuaciones estructurales, Mínimos cuadrados parciales

\section{Códigos JEL: I23}


Title: Acceptance and use of e-learning systems by undergraduate students of Ecuador: The case of a state university

\section{Abstract}

Purpose: The purpose of this research was to adapt the Technology Acceptance Model (TAM) to predict the use and intended use of e-learning systems among undergraduate students at a state university in Ecuador, with the intention of improving the understanding of those factors that could enhance the utilization of these systems and the implementation of policies to increase the benefits they bring to the process of teaching and learning.

Design/methodology: The analysis has epistemological basis on the empirical-inductive, based on observation of perception. Based on the literature review on the evaluation and acceptance of e-learning systems, the model has been adapted to the case of Ecuador and a questionnaire with 52 items based on Likert scales was developed. The instrument was emailed to 600 undergraduate students from a state university in Ecuador, being 423 of them answered satisfactorily. We performed validity and reliability tests of structural equation model by partial least squares (PLS), with the support of statistical software SmartPLS.

Contributions and results: The results indicate that all the hypotheses of TAM are met in the Ecuadorian context, when assessing the acceptance of e-learning systems among undergraduate students at a state university. The main contribution was to identify that the perceived enjoyment, social influence and computer self-efficacy have a direct effect on the two main constructs of TAM, perceived ease of use (PEOU) and perceived usefulness (PU). It was also found that satisfaction (S) is highly influenced by PU and S influences the use of e-learning systems, being the first a specific contribution of this study. Technical support showed no influence on the fundamental constructs of TAM.

Limitations: The research was conducted to undergraduate students in one state Category B university of Ecuador, being the reality of this country more complex, as there are four categories with different characteristics. We haven't considered neither age, gender, graduate students nor socioeconomic status, among other student characteristics that may affect the investigation.

Practical implications: Identifying the factors that influence the acceptance and use of elearning systems will help to create and improve teaching and learning environments for 
undergraduate students. Allowing exploiting the benefits of these technological tools more efficiently, adapting them to management policies of the institutions of higher education.

Originality/value: This paper presents empirical data on the use and acceptance of e-learning systems for undergraduate students of a particular university in Ecuador. No similar studies are evident in the country and the model can be considered for future studies of national scope.

Keywords: e-learning, TAM, PLS, SEM, Structural equation models, Partial least squares

Jel Codes: I23

\section{Introducción}

Desde los años setenta se ha tratado de incorporar sistemas de información con la finalidad de mejorar la productividad de las organizaciones. Las Tecnologías de la Información y la Comunicación (TIC) facilitan el procesamiento y distribución de información de datos vía electrónica (Lobato Rubio, 2008).

Ante las bondades que brinda el desarrollo de las TIC en el ámbito educacional, surgen sistemas e-learning con mayores potencialidades, que proporcionan nuevas formas de diseñare impartir educación (Mallak, 2001; University of Oxford International Strategy Office, 2015), y que pueden cambiar la forma en que funcionan los procesos, en relación a cómo aprenden los estudiantes y cómo enseñan los profesores (Landry, Griffeth \& Hartman, 2006).

Los sistemas e-learning vinculan nuevas formas y estrategias de aprender mediante el uso de las TIC, tanto en las instituciones de educación superior como en la formación corporativa (Y.H. Lee, Hsieh, \& Hsu, 2011; Melas, Zampetakis, Dimopoulou \& Moustakis, 2011). Se utiliza tecnología en el proceso de enseñanza-aprendizaje para contribuir en gran medida, a que las universidades alcancen el papel de agente y agencia del desarrollo científico tecnológico de los países.

El uso de la tecnología dio origen a investigaciones para evaluar su aceptación, tomando mayor énfasis a mediados de los ochenta. Entre las principales teorías que se identificaron para evaluar la aceptación de los sistemas de información en general y que también han sido aplicadas para evaluar la aceptación de los sistemas e-learning, tenemos: el TAM, modelo de aceptación de la tecnología (Davis, 1985); UTAUT, teoría unificada de aceptación y uso de la tecnología (Venkatesh, Morris, Davis \& Davis, 
2003); 3-TUM, modelo de tres niveles del uso de la tecnología (Liaw, Chang, Hung \& Huang, 2006); TTF, modelo de ajuste de tareas tecnológicas (Dishaw \& Strong, 1999); TPB, modelo teoría de la conducta planificada (Fu, Farn \& Chao, 2006).

A pesar de los múltiples beneficios demostrados por la utilización de los sistemas e-learning a menudo, y también en el contexto universitario,éstos no se hacen efectivos. Bien sea por no alcanzarse los objetivos planteados en el proceso de enseñanza-aprendizaje (Chen, 2011), o bien sea por la poca aceptación que estas herramientas puedan tener entre los estudiantes. En consecuencia, parece oportuno que las universidades se esfuercen en entender mejor la relación entre los sistemas e-learning y el proceso de enseñanza-aprendizaje, así como,en mejorar su conocimiento sobre los factores que pueden potenciar y mejorar el nivel de aceptación y uso de estos sistemas (Parker, 1999).

Por otra parte, Internet es una tecnología global y ampliamente utilizada, pero su eficacia se debe medir a nivel local, puesto que los usuarios a menudo trabajan en contextos locales o nacionales (Li \& Kirkup, 2007). Por ello, procede investigar la aceptación de los sistemas e-learning entre los estudiantes en el caso específico de Ecuador, y más cuando se constata que existen pocos estudios de este tipo en el contexto de América Latina, dado que los factores culturales varían de un país a otro y entre los estudiantes universitarios esto no es la excepción.

Entre las opciones existentes para comprender el proceso de aceptación de los sistemas de información por parte de los usuarios, el TAM es uno de los marcos teóricos más citados por la literatura académica (Mirriahi, Unit, Vaid \& Burns, 2015; Park, Lee \& Cheong, 2007; Ramirez-Correa, Javier RondanCataluña, Arenas-Gaitán \& Alfaro-Perez, 2016; Šumak, Heričko \& Polančič, 2011). Se trata de un modelo consolidado que se ha mostrado útil, y para el cual existe todavía interés en revisarlo para ampliarlo o modificarlo de acuerdo a los rápidos cambios en las tecnologías y sus entornos (Kripanont, 2006). Por estas razones nuestro estudio se basó en una adaptación de este modelo.

En el contexto expuesto,el objetivo de esta investigación fue adaptar el modelo TAM para predecir el uso y la intención de uso de los sistemas e-learning entre los estudiantes de grado de una universidad estatal de Ecuador, en vistas a obtener un mejor entendimiento de los factores que condicionan su utilización y poder aplicar políticas adecuadas para mejorar los beneficios que aportan en el proceso de enseñanza y aprendizaje.

Para alcanzar estos objetivos se ha adaptado el modelo TAM al caso de Ecuador y se ha realizado un análisis basado en la observación de la percepción a partir de la elaboración de un cuestionario que fue enviado a una muestra de estudiantes de grado de una universidad estatal en Ecuador, siendo utilizado 
para el análisis empírico un modelo de ecuaciones estructurales (SEM) basado en mínimos cuadrados parciales (PLS).

En el segundo apartado se revisa la literatura relativa a los sistemas e-learning y a los modelos de aceptación de la tecnología. El modelo y las hipótesis se tratan en el tercer apartado, siendo tratada la metodología en el cuarto. Los resultados se presentan en el quinto apartado y la discusión, los futuros estudios y las conclusiones configuran el sexto y último apartado.

\section{Revisión de la literatura}

\subsection{Sistema e-learning}

Los sistemas de gestión del aprendizaje como también se conoce a los sistemas e-learning, son un tipo de aplicación de software para dar soporte a los cursos presenciales, en línea o mixtos. Proporcionan herramientas y características tales como: administración, comunicación síncrona y asíncrona, compartición multimedia, evaluación y seguimiento, y de compatibilidad estándar (Tawfik et al., 2013).

Los sistemas e-learning permiten continuar las clases sin restricciones asociadas al tiempo y espacio. Ayudan a mejorar la colaboración e interactividad entre las personas que aprenden y las personas que enseñan (Adam, Vallés \& Rodríguez, 2013). Y pueden ser interpretados de varias maneras, tales como, "sistemas de suministro de la educación basado en ordenador que se proporciona a través de Internet", o "un método educativo que es capaz de ofrecer oportunidades a las personas necesarias, en el lugar correcto, con los contenidos adecuados, y el momento adecuado” (Lee \& Lee, 2008).

Muchas instituciones están incorporando sistemas e-learning en sus procesos formativos para de esta manera mejorar sus actividades. Esta forma de aprendizaje depende actualmente de las redes y ordenadores pero es probable que vayan evolucionando hacia sistemas que comprendan una variedad de canales, por ejemplo: TV por cable, satélites, teléfonos móviles y otras tecnologías (Caporarello \& Sarchioni, 2014). 


\subsection{Modelo de Aceptación de la Tecnología (TAM)}

Desde mediados los años ochenta, la medición de la aceptación de la tecnología fue un área de investigación que recibió una destacada atención por parte de los investigadores. En 1986 se propuso el modelo TAM (Legris, Ingham \& Collerette, 2003), el cual se basa en la teoría de la acción razonada (Fishbein \& Ajzen, 1975).

La actitud general de un usuario hacia el uso de un posible sistema dado, tal como la WWW, se muestra como una función de los constructos de creencias en el TAM: Utilidad Percibida y Facilidad de Uso Percibida (Feneche, 1998).

Posteriormente se mejoró el modelo creándose su segunda versión, TAM2 (Davis, Bagozzi \& Warshaw, 1989; Venkatesh \& Davis, 1996). El desarrollo del TAM2 incluiría la intención de uso como una nueva variable directamente influenciada por la utilidad percibida de un sistema (Bagozzi, 2007; Davis, 1989). El modelo TAM2 se muestra en la Figura 1.

TAM2 postula que las variables externas intervienen indirectamente influyendo en la Utilidad Percibida (PU) y la Facilidad de Uso Percibida (PEOU); se considera que este tipo de variables pueden influir en los constructos PU y PEOU, y a su vez en los constructos relativos al uso de sistema (Legris et al., 2003), en el cual la utilidad percibida y la percepción de la facilidad de uso ya jugaban un papel importante en las decisiones que afectan la adopción de tecnología (Liao \& Lu, 2008).

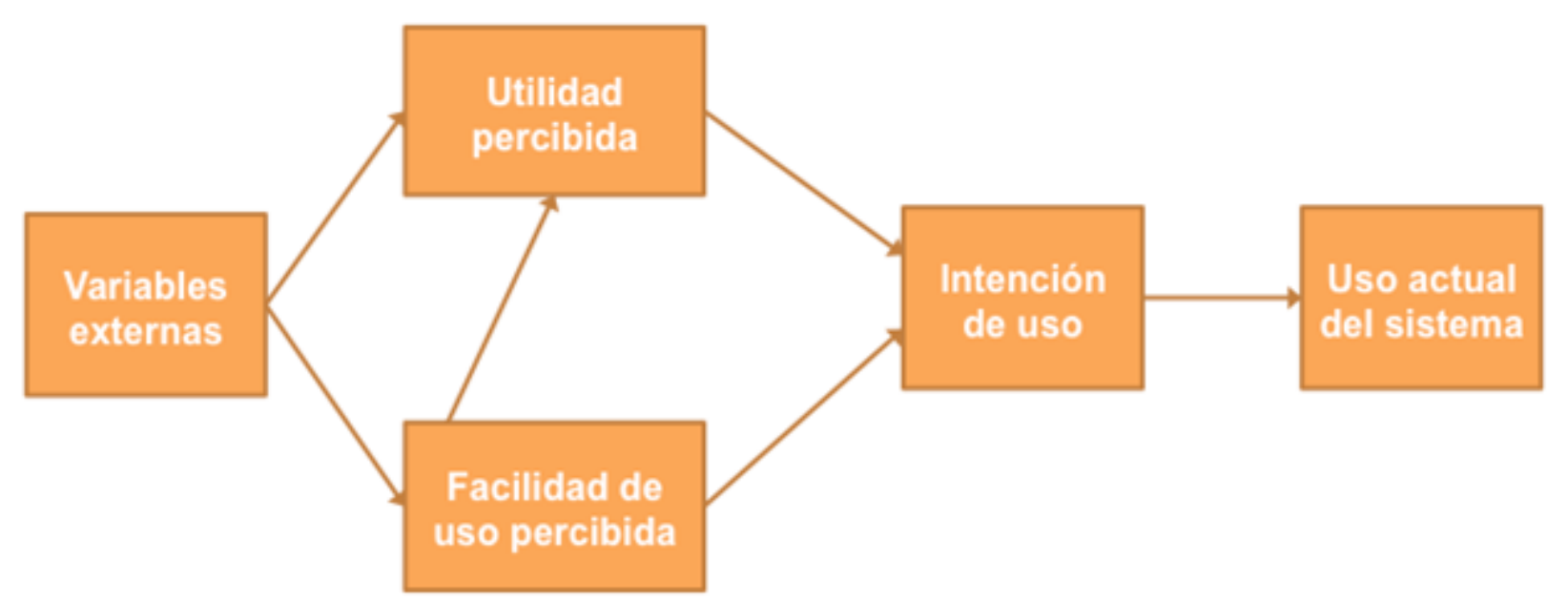

Figura 1. TAM 2 
Venkatesh y Bala (2008) combinaron TAM2 y el modelo de los determinantes de la facilidad de uso percibida (Venkatesh, 2000), y desarrollaron un modelo integrado de aceptación de la tecnología, el TAM3. Este modelo es una red nomológica completa de los determinantes de adopción y uso de TIC de los individuos. Sugirieron tres extensiones teóricas más allá del TAM2 y el modelo de los determinantes de la facilidad de uso percibida.

La extensión referida en el párrafo anterior consistió en ampliar el número de determinantes que afectan a la utilidad percibida y la facilidad de uso percibida de una innovación. Los factores que influyen en la utilidad percibida son norma subjetiva, imagen, pertinencia del trabajo, calidad de salida, y demostrabilidad del resultado. La facilidad de uso percibida está influenciada por variables de anclaje (auto-eficacia computacional, las percepciones de control externo, la ansiedad por ordenador, el disfrute por ordenador) y las variables de ajuste (Disfrute percibido y la usabilidad Objetiva). La experiencia y la voluntariedad actúan como modificadores de la intención de conducta (Jeffrey, 2015).

El TAM y sus versiones han dado pauta para el desarrollo de otras investigaciones que han permitido: la réplica de TAM, las pruebas de sus proposiciones y las posibles limitaciones; la comparación de TAM con otros modelos como la teoría de la conducta planificada (TPB) (Humaidi, 2013; Williams, Nripendra \& Dwived, 2015); la adaptación de TAM para diversas configuraciones tales como escenarios obligatorios, diferentes aplicaciones y culturas; y,extensión del modelo para incluir otras variables (Šumak, Heričko \& Polančič, 2011), como es el caso del presente estudio.

Como opción al TAM, surgen otras teorías ya citadas que también pueden ser utilizadas y/o ampliadas para propósitos similares y que se describen brevemente a continuación.

UTAUT es el modelo de la teoría unificada de aceptación y uso de la tecnología, proporciona una herramienta útil para los administradores que necesitan evaluar la probabilidad de éxito, para la introducción a nuevas tecnologías y les ayuda a entender las causas de la aceptación a fin de diseñar intervenciones de forma proactiva. Está dirigido a los usuarios que pueden estar menos inclinados para adoptar y usar nuevos sistemas. El modelo fue propuesto por Viswanath Venkatesh et al. (2003), explica la intención del usuario para utilizar la tecnología y el comportamiento de su uso.

3-TUM es el modelo de tres niveles del uso de la tecnología, fue desarrollado por Liaw, Huang y Chen (2007). Se define como perspectivas multidisciplinares que comprenden la motivación, la teoría cognitiva social, la teoría de conducta planificada y el TAM (Tsai, Chuang, Liang \& Tsai, 2011). De acuerdo con el modelo 3-TUM, las actitudes individuales hacia la tecnología se pueden dividir en los 
siguientes niveles: de la experiencia individual y la calidad del sistema, afectivo y cognitivo, intención de comportamiento (Šumak, Heričko \& Polančič, 2011).

TTF es el modelo de ajuste de tareas-tecnológicas, es utilizado para medir el grado en que una tecnología ayuda a un individuo en el desempeño de su portafolio de tareas (Mcgill, Klobas \& Renzi, 2011). Se considera que el modelo TTF puede ser útil en los sistemas e-learning para dar soporte al instructor en el rango de la enseñanza y las actividades de administración del curso.

Šumak, Heričko and Polančič (2011) realizaron un meta análisis a 42 estudios de la aplicación del TAM en el contexto del e-learning, en él se identificó que el $86 \%$ de los trabajos utilizaron el TAM como su marco de referencia. Los estudios examinados incluyeron empleados, profesores y estudiantes. Por este motivo, sumado a lo ya revisado en este apartado se consideró factible utilizar el TAM como modelo base para la presente investigación.

\section{Modelo e hipótesis de investigación}

Al examinar la evolución del modelo TAM en la literatura aplicada a la evaluación de la aceptación de los sistemas e-learning (Kripanont, 2006; Legris et al., 2003; Šumak, Heričko, Pušnik \& Polančič, 2011), se eligieron diversos constructos e ítems, que mejor se adaptaban al contexto ecuatoriano.

La adaptación del modelo consistió en una traducción de estudios previos, y su revisión por cuatro profesores expertos de Venezuela, Colombia, Ecuador y España. La adaptación fue necesaria, ya que anteriormente se realizaron estudios en contextos culturales diferentes al latinoamericano y específicamente al ecuatoriano. Los profesionales que revisaron la adaptación, tienen conocimiento del contexto ecuatoriano, por ello el aporte que dieron en cuanto a la adaptación del modelo.

Como punto de partida los expertos recibieron un formulario con el modelo inicial y los indicadores identificados como pertinentes en la revisión de la literatura; luego se aplicó una triangulación con sus respuestas, de manera que se aceptaron las recomendaciones que coincidieron u otras que se consideraron especialmente adecuadas. De este proceso resultaron cinco constructos adicionales a los definidos en el modelo TAM2 estándar y una lista de indicadores.

Las hipótesis se presentan y se describen en los apartados siguientes. 


\subsection{La influencia social}

Se define como el cambio en los pensamientos, sentimientos, actitudes o el comportamiento de un individuo que resulta de la interacción con otra persona o grupo (Kocaleva, 2015). Se incluyó este constructo en la investigación considerando que el aspecto que evalúa, coincide con el comportamiento latinoamericano y en especial de Ecuador. Estudios previos antes analizaron este factor como importante (Abbad, Morris \& de Nahlik, 2009; Chou \& Tseng, 2011; Udzlmd, Bachtiar, Rachmadi \& Pradana, 2014).

La influencia social, es el proceso por el cual las personas hacen cambios reales a sus sentimientos y comportamientos como resultado de la interacción con otras personas que son percibidas como similares, deseables o expertos. (Rashotte, 2007). La hipótesis que se planteó respecto a este constructo es la siguiente:

\section{H1. La influencia social tiene un impacto positivo en la utilidad percibida.}

\subsection{El entretenimiento percibido}

El entretenimiento percibido es el grado en que la actividad de la utilización de la tecnología se percibe como agradable en su propio derecho, además de las consecuencias de rendimiento que pueden ser anticipados (Padilla-Meléndez, Águila-obra \& Garrido-Moreno, 2015). Davis, Bagozzi y Warshaw (1992), proponen el modelo TAM incluyendo el disfrute percibido como factor de motivación intrínseca.

Por la aparición de múltiples bondades tecnológicas y que su uso debe ir acompañado de un disfrute o entretenimiento percibido, se decidió incorporar este constructo en la investigación. Para ello también se evaluaron estudios previos al respecto (Duenas-Rugnon, Iglesias-Pradas \& Hernandez-Garcia, 2010; Elkaseh, Wong \& Fung, 2015; Duenas-Rugnon et al., 2010; Elkaseh et al., 2015). Las hipótesis que corresponde a este constructo son las siguientes:

H2.1. El entretenimiento percibido tiene un impacto positivo en la utilidad percibida.

H2.2. El entretenimiento percibido tiene un impacto positivo en la facilidad de uso percibida. 


\subsection{Soporte técnico}

El soporte técnico se refiere a las personas capacitadas para ayudar a los usuarios en la solución de problemas relacionados con el hardware y software de computadora, para lo cual se valen de múltiples opciones que les permite interactuar de forma directa (Arteaga \& Duarte, 2010).

La disponibilidad de apoyo técnico es uno de los factores importantes en la determinación de la aceptación de la tecnología para la enseñanza (Abbad et al., 2009), especialmente en la etapa de inicio de la adopción de la tecnología. Lo expuesto coincide con los resultados de varios estudios (AlQudah Ahmed, 2014; Arteaga \& Duarte, 2010; Ngai, Poon \& Chan, 2007; Ramirez-Anormaliza, Sabaté \& Guevara-Viejo, 2015). Esto motivó la inclusión de este constructo en la presente investigación. Las hipótesis que corresponden a este constructo son las siguientes:

\section{H3.1. El soporte técnico tiene un impacto positivo en la utilidad percibida.}

\section{H3.2. El soporte técnico tiene un impacto positivo en la facilidad de uso percibido.}

\subsection{Auto-eficacia computacional}

Es un juicio individual de la eficacia a través de múltiples dominios de computadoras y aplicaciones específicas. La auto-eficacia es definidacomo una percepción individual de la eficacia en el uso de una aplicación o sistema específico dentro del dominio de la informática en general (Hsiao, Wu \& Chen, 2013; Yi \& Hwang, 2003).

La auto-eficacia computacional es un concepto importante en la teoría del aprendizaje social (Bandura, 1977). Varios estudios han encontrado que las percepciones de auto-eficacia pueden influir en las decisiones acerca de qué comportamientos se pueden llevar a cabo (Brown \& Inouye, 1978; Wood \& Bandura, 1989).

En el contexto de e-learning, la auto-eficacia computacional se interpreta, desde la perspectiva de un estudiante, como la auto-confianza en su o sus habilidades para realizar ciertas tareas de aprendizaje al usar un sistema de gestión de aprendizaje. Se considera que un estudiante que tiene un fuerte sentido de su capacidad para hacer frente a este tipo de sistemas, puede tener una percepción muy positiva de su facilidad de uso y la utilidad. Por lo tanto es probable que esté más dispuesto a aceptar y utilizar la herramienta (Abbad et al., 2009). Las hipótesis que corresponden a este constructo son las siguientes: 
H4.1. La auto-eficacia computacional tiene un impacto positivo en la utilidad percibida.

H4.2. La auto-eficacia computacional tiene un impacto positivo en la facilidad de uso percibida.

\subsection{Utilidad percibida y facilidad de uso percibida}

La utilidad percibida (PU) y la facilidad de uso percibida (PEOU) tienen influencia significativa sobre la intención de un individuo en el uso de las tecnologías (Ma \& Liu, 2004). PU y PEOU son constructos básicos del TAM. También se han identificado estudios donde PU tiene influencia sobre la satisfacción y ésta,a su vez, sobre la intención de uso (Calli, Balcikanli, Calli, Cebeci \& Seymen, 2013; Chih-Yang, Tsai-Chu, Ping-Teng \& Chih-Wei, 2011; Cho, Cheng \& Hung, 2009; J.W. Lee, 2010; T.-C. Lin \& Chen, 2012; Sahin \& Shelley, 2008). Por lo tanto, fueron incluidos en la presente investigación hipótesis asociadas a dichos constructos y son las siguientes:

H5.1. La facilidad de uso percibida tiene un impacto positivo en la utilidad percibida.

H5.2. La facilidad de uso percibida tiene un impacto positivo en la intensión de uso.

H6.1. La utilidad percibida tiene un impacto positivo en la satisfacción.

H6.2. La utilidad percibida tiene un impacto positivo en la intensión de uso.

\subsection{Satisfacción}

En el contexto objeto de estudio, la satisfacción del usuario es el grado en que el sistema e-learning cumple con sus necesidades de información (Rey Martín, 2000). Por lo tanto se consideró que si los estudiantes perciben como útil el sistema e-learning, esto puede influenciar en la satisfacción y por ende los estudiantes tenderían a utilizarlo. La hipótesis asociada a este constructo es la siguiente:

H7.0. La satisfacción tiene un impacto positivo en el uso del sistema.

Hay antecedentes de estudios previos que apoyan el planteamiento de las hipótesis 6.1 y 7.0 (Antón, Camarero \& San José, 2013; M.-C. Lee, 2010; Weng \& Tsai, 2015), por ello fueron evaluadas en esta investigación. Estos estudios sostienen que la satisfacción de un usuario respecto a una tecnología 
específica se ve influenciada por la utilidad percibida y a su vez la satisfacción influye directamente en el uso de la misma.

\subsection{Intención hacia el uso}

Es el grado en el cual una persona tiene planes conscientes formulados para llevar a cabo, o no, alguna conducta específica (Findik \& Kunçay, 2009; Little, 2009; Šumak, Heričko \& Polančič, 2011; Welch, Ray $\&$ Peterson, 2015). Este constructo es parte estructural del TAM,por ello se incluyó en este estudio y la hipótesis asociada es la siguiente:

H8.0. La intención hacia el uso tiene un impacto positivo en el uso del sistema.

La relación entre las cinco variables externas que se presentan y PU - PEOU como marco de estudio se muestran en la de la sección diseño y metodología. Las hipótesis 5.1, 5.2, 6.2, 7.0 y 8.0 fueron planteadas para mejorar la comprensión del modelo que se aplicó en este estudio, las mismas fueron confirmadas en toda la literatura revisada.

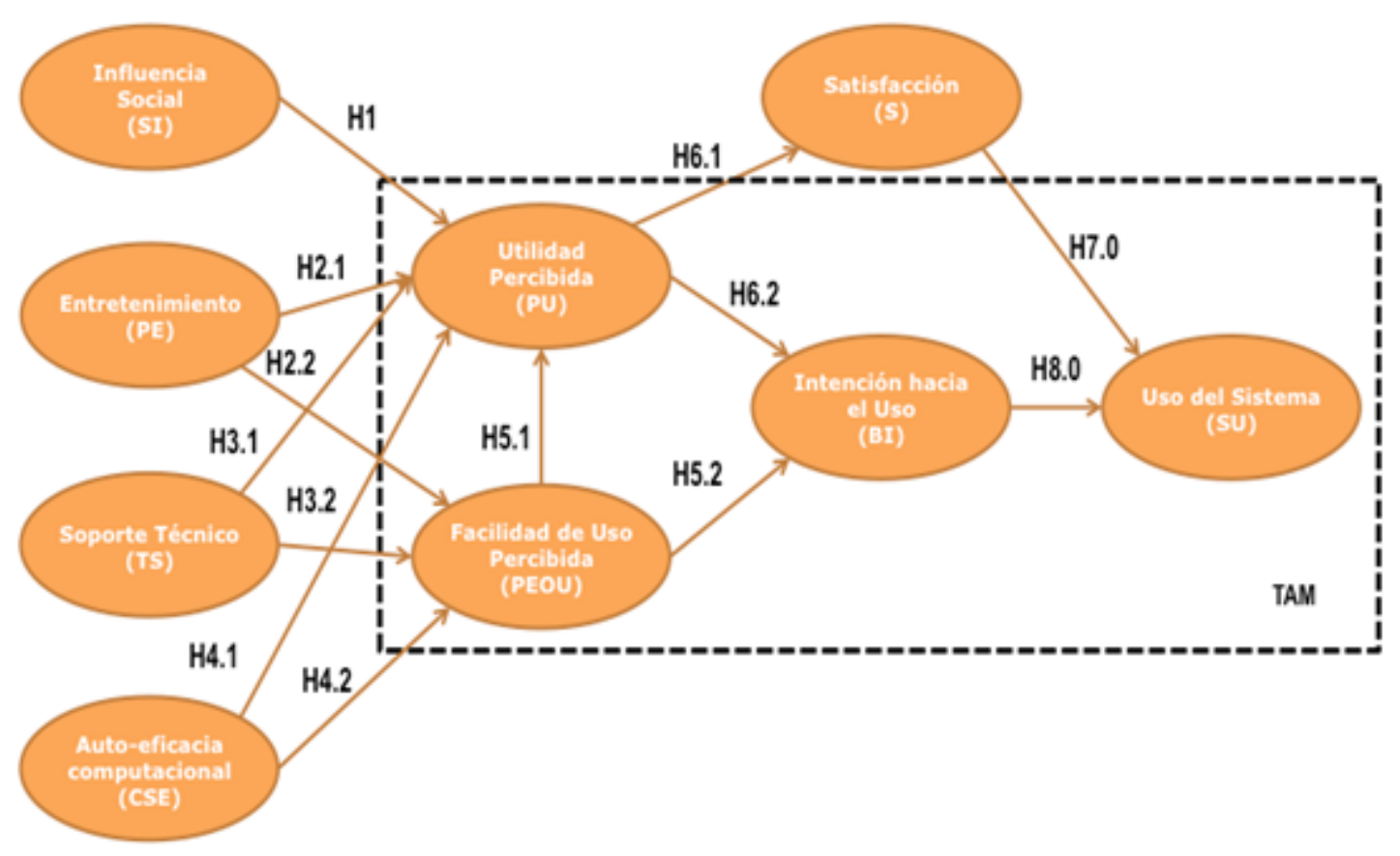

Figura 2. Modelo de investigación 


\section{Diseño/metodología}

\subsection{Recolección de datos y características de la muestra}

En Ecuador, las universidades se encuentran clasificadas en cuatro categorías (A, B, C y D) según su desempeño en docencia e investigación (CONEA, 2009). La encuesta, que incluía 52 ítems, se aplicó a 600 estudiantes de grado de una universidad estatal en Ecuador perteneciente a la categoría B, durante el año 2015.

Un total de 423 encuestas fueron finalizadas satisfactoriamente, lo cual indicó una tasa de respuesta del 71\%. De la muestra, 174 eran hombres y 249 mujeres; 98 estudiantes eran menores de 20 años, 274 tenían entre 20 y 30 años, 31 entre 30 y 40 años y por último, 20 estudiantes tenían más de 40 años de edad. Del total de encuestados, 86 estudiantes trabajaban a tiempo parcial, 76 a tiempo completo y 261 no trabajaban. En la Tabla 1 se muestran los diferentes grupos de estudiantes divido por género, edad, ocupación y otros datos demográficos.

\begin{tabular}{|l|l|c|r|}
\hline \multicolumn{2}{|c|}{ Característica } & Frecuencia & Porcentaje \\
\hline \multirow{4}{*}{ Género } & Masculino & 174 & 41.13 \\
\cline { 2 - 4 } & Femenino & 249 & 58.87 \\
\hline \multirow{5}{*}{ Edad } & Menos de 20 & 98 & 23.17 \\
\cline { 2 - 4 } & Entre 20 y 30 & 274 & 64.78 \\
\cline { 2 - 4 } & Entre 30 y 40 & 31 & 7.33 \\
\cline { 2 - 4 } & Más de 40 & 20 & 4.73 \\
\hline \multirow{5}{*}{ Ocupación } & Medio tiempo & 86 & 20.33 \\
\cline { 2 - 4 } & Tiempo completo & 76 & 17.97 \\
\cline { 2 - 4 } & No trabaja & 261 & 61.70 \\
\hline \multirow{2}{*}{ Internet en casa } & Si & 341 & 80.61 \\
\hline & No & 82 & 19.39 \\
\hline \multirow{5}{*}{ Experiencia en internet } & Si & 339 & 80.14 \\
\cline { 2 - 4 } & No & 50 & 19.86 \\
\hline & Entre 1 y 2 años & 299 & 11.82 \\
\cline { 2 - 4 } & Más de 2 años & 23 & 70.69 \\
\cline { 2 - 4 } & Menos de 1 año & & 12.06 \\
\cline { 2 - 4 } & Nunca & 5.44 \\
\hline
\end{tabular}

Tabla 1. Estadística de las características demográficas de los encuestados

Los estudiantes encuestados utilizan el sistema e-learning Moodle. Su participación fue voluntaria y no se ofreció ningún incentivo financiero. Para la gestión de la encuesta se utilizó un sistema web propio de la misma universidad. 
Siguiendo la pauta marcada en investigaciones anteriores y similares a ésta, se utilizó la técnica de ecuaciones estructurales, basada en un enfoque mínimos cuadrados parciales (PLS). Esta técnica relaciona las variables latentes (constructos o variables que no son directamente observables), con indicadores o elementos que son directamente observables (Schumacker \& Lomax, 2010). Para este fin se utilizó el software SmartPLS versión 3.2.4 (Garson, 2016).

Los ítems se adaptaron tomando como referencia estudios anteriormente publicados en revistas académicas. Se midieron en una escala tipo Likert de siete puntos, donde los encuestados debían indicar el grado en que estuvo de acuerdo con una determinada declaración, que van desde totalmente en desacuerdo (1) a totalmente de acuerdo (7).Los ítems que se utilizaron en la investigación se presentan en el Anexo 1.

\section{Resultados}

PLS es una técnica SEM basada en la varianza, es ampliamente utilizada en administración y las ciencias sociales. Su capacidad de modelar factores hace que sea considerada como una buena opción de herramienta estadística, para la investigación de nuevas tecnologías (Henseler, Hubona \& Ray, 2016). Por lo tanto fue la elegida para esta investigación.

La evaluación del ajuste del modelo se realizó en dos etapas (Hulland, 1999). En primer lugar, se evaluó el modelo de medición (validez de constructo y la fiabilidad de las medidas); en segundo lugar, el modelo estructural (la prueba de las hipótesis). Posteriormente se evaluó la validez predictiva del modelo.

\subsection{Evaluación del modelo de medidas}

Para verificar la validez y la fiabilidad de las medidas, el factor de carga fue observado a partir del análisis factorial confirmatorio (CFA), considerando el modelo de medición bajo el enfoque PLS (Garson, 2016). También fueron examinados la fiabilidad compuesta y la varianza media extraída (AVE). El factor de carga del CFA proporciona evidencia de validez convergente, siendo todos los ítems de carga suficientemente altos en los constructos correspondientes. Todos ellos superan el valor límite de 0.85 sugerido por Peterson (2000). 
Para el análisis de fiabilidad se evaluó la fiabilidad compuesta, que en todos los casos calculaban entre 0.95 y 0.98. Las medidas de ajuste correspondiente se pueden encontrar en el Anexo 2. Para comprobar la validez discriminante, se aplicó la prueba de Fornell \& Larcker (1981). El procedimiento dicta que la raíz cuadrada de la AVE de cada constructo supera la correlación compartida entre el constructo y otros constructos en el modelo, con el fin de obtener la validez discriminante. La Tabla 2 muestra los detalles de este análisis. Todos los constructos superaron con éxito la prueba; la raíz cuadrada del AVE (en diagonal) es mayor que las correlaciones cruzadas con otros constructos. Todos los criterios de elegibilidad exceden los niveles de límite comúnmente sugeridos en la literatura y muestran una buena fiabilidad y validez de todos los constructos.

Para una buena fiabilidad, se deben considerar los ítems con cargas factoriales superiores a 0.707 (Carmines \& Zeller, 1994); constatándose que todos los ítems en este estudio cumplieron esa condición.

\begin{tabular}{|l|c|c|c|c|c|c|c|c|c|}
\cline { 2 - 11 } \multicolumn{1}{c|}{} & BI & CSE & PE & PEOU & PU & S & SI & SU & TS \\
\hline BI & 0.942 & & & & & & & & \\
\hline CSE & 0.849 & 0.900 & & & & & & & \\
\hline PE & 0.844 & 0.757 & 0.901 & & & & & & \\
\hline PEOU & 0.872 & 0.843 & 0.772 & 0.961 & & & & & \\
\hline PU & 0.913 & 0.846 & 0.863 & 0.854 & 0.956 & & & & \\
\hline S & 0.913 & 0.828 & 0.888 & 0.850 & 0.929 & 0.955 & & & \\
\hline SI & 0.759 & 0.770 & 0.776 & 0.721 & 0.800 & 0.777 & 0.897 & & \\
\hline SU & 0.852 & 0.786 & 0.763 & 0.798 & 0.831 & 0.826 & 0.723 & 0.917 & \\
\hline TS & 0.656 & 0.642 & 0.686 & 0.594 & 0.668 & 0.696 & 0.645 & 0.672 & 0.892 \\
\hline
\end{tabular}

Tabla 2. Validez discriminante y convergente de los constructos

\subsection{Evaluación del modelo estructural}

La significancia estadística de las relaciones en el modelo estructural fue evaluada mediante SmartPLS 3.2.4 (Garson, 2016). Para el efecto se utilizó el método de remuestreo no paramétrico bootstrap (1000 submuestras) desarrollado por Badley Efron (Gómez Cruz, 2011).

Tal y como muestra la Figura 3, se encontró que la utilidad percibida y la facilidad de uso percibida tienen un impacto positivo directo en la intención hacia el uso. El entretenimiento percibido, la facilidad de uso percibida, la influencia social y la auto-eficacia computacional tienen un impacto positivo directo en la utilidad percibida. El entretenimiento percibido y la auto-eficacia computacional tienen un impacto positivo directo en la facilidad de uso percibida. La intención hacia el uso del sistema y la 
satisfacción tienen un impacto positivo directo en el uso del sistema. La utilidad percibida tiene un impacto directo positivo muy alto en la satisfacción. El estudio evidencia el rechazo de la hipótesis del impacto de soporte técnico sobre la facilidad de uso percibida y sobre la utilidad percibida.

PLS no tiene medida de bondad de ajuste única, ya que su principal objetivo es la maximización de la varianza explicada. La calidad de un modelo PLS puede determinarse mediante el examen de los valores de R2 de los constructos endógenos (Hulland, 1999). El modelo explica el 74\% de varianza en SU, 87\% de la varianza en BI, 86\% de la varianza en S, 86\% de la varianza en PU y 75\% de la varianza en PEOU.

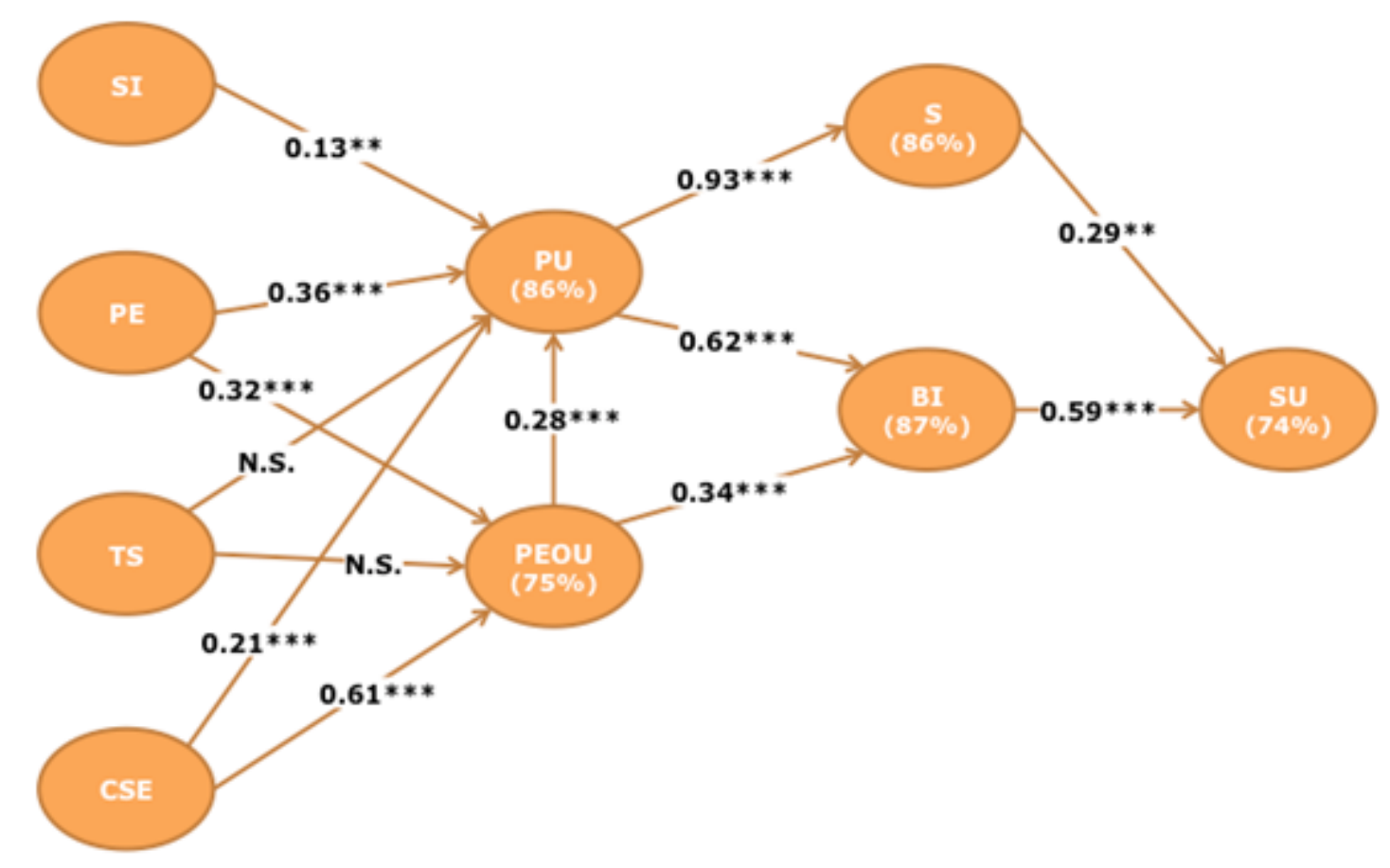

Figura 3. Resultados PLS. Notas: Varianza explicada $\left(\mathrm{R}^{2}\right)$ entre paréntesis. ${ }^{*}$ Coeficiente de ruta significativa al nivel de 0.05 ; ** $\mathrm{Al}$ nivel 0.01 ; *** al nivel 0.001

Los datos procesados sirven como indicadores de la capacidad explicativa del modelo, estos permiten rechazar las hipótesis 3.1 y 3.2, así como aceptar al resto de hipótesis planteadas. Con ello se confirman las hipótesis del TAM en el contexto ecuatoriano, entre los estudiantes de grado de una universidad pública, en el uso de los sistemas e-learning. Los resultados aportan los constructos SI, PE y CSE como variables externas influyentes en la aceptación y uso de los sistemas e-learning en el caso de Ecuador, 
constructos que se pueden incluir en un estudio futuro a nivel nacional. De igual manera este estudio sugiere incorporar el constructo S, como se puede ver en el modelo de la Figura 3.

Con lo expuesto en el párrafo anterior se logra identificar como factores clave para potenciar el uso de los sistemas e-learning entre los estudiantes de grado a los constructos SI, PE y CSE.

\subsection{Evaluación de la validez predictiva del modelo}

El análisis mostró la relevancia predictiva y validez del modelo para evaluar la aceptación de los sistemas e-learning, entre los estudiantes universitarios de grado en Ecuador. La relevancia predictiva está dada por el resultado del índice Stone-Geisser's Q² (Garson, 2016) en los constructos endógenos, el mismo que debe ser mayor que cero. Para este estudio en todos los casos se cumplió ésta condición, tal como se observa en la Tabla 3, donde se muestra la redundancia de constructo validada de forma cruzada. Por lo tanto el modelo tiene relevancia predictiva.

\begin{tabular}{|l|r|}
\hline \multicolumn{1}{|c|}{ Constructo } & $\mathbf{Q}^{\mathbf{2}}$ \\
\hline BI & 0.77 \\
\hline PEOU & 0.70 \\
\hline PU & 0.78 \\
\hline S & 0.79 \\
\hline SU & 0.62 \\
\hline
\end{tabular}

Tabla 3. Redundancia de constructo validada de forma cruzada

(Cohen, 1988) sostiene que para determinar el nivel predictivo del modelo se puede considerar a 0.02 como pequeño, 0.15 representa un tamaño medio, y 0.35 representa un tamaño alto.

Los resultados obtenidos dan pauta para mejorar el modelo. Con esta finalidad se evaluaron las siguientes acciones: eliminar el constructo soporte técnico (TS), por cuanto no evidencia influencia sobre los constructos fundamentales del TAM (sus hipótesis asociadas resultaron no significativas); eliminar los ítems con menor carga factorial en los constructos que tienen 6 o más ítems para de tener un cuestionario menos denso (S1, SU2, CSE2, CSE5, CSE6 y CSE9); y, eliminar el constructo S.

El proceso descrito en el párrafo anterior permitió construir la Tabla 4, donde se resumen los principales índices de calidad de un modelo PLS. Estos son: el de ajuste global (SRMR), R2 y el de 
relevancia predictiva Q2. Con este análisis se sugiere que para estudios futuros es válido omitir el constructo TS y reducir elnúmero de indicadores como se expuso en el párrafo anterior, ya que los índices de calidad del modelo no se afectan, así mismo no se recomienda eliminar el constructo $\mathrm{S}$ ya que el hacerlo afecta a 5 de los índices evaluados.

\begin{tabular}{|c|c|c|c|c|c|}
\hline Criterio & $\begin{array}{c}\text { Índice / } \\
\text { Constructo }\end{array}$ & Modelo Original & $\begin{array}{c}\text { Reducir } \\
\text { indicadores }\end{array}$ & $\begin{array}{c}\text { Eliminar } \\
\text { constructo TS }\end{array}$ & $\begin{array}{c}\text { Eliminar } \\
\text { Constructo S }\end{array}$ \\
\hline Ajuste global & SRMR & 0.037 & $\underline{0.034}$ & 0.032 & $\underline{0.033}$ \\
\hline Calidad R ${ }^{2}$ & $\mathrm{BI}$ & 0.865 & $\overline{0.865}$ & $\overline{0.865}$ & $\overline{0.865}$ \\
\hline Calidad $\mathrm{R}^{2}$ & PEOU & 0.753 & $\underline{0.748}$ & $\underline{0.748}$ & $\underline{0.748}$ \\
\hline Calidad $\mathrm{R}^{2}$ & $\mathrm{PU}$ & 0.859 & 0.860 & 0.860 & 0.860 \\
\hline Calidad $\mathrm{R}^{2}$ & S & 0.863 & 0.864 & 0.864 & \\
\hline Calidad $\mathrm{R}^{2}$ & SU & 0.740 & 0.741 & 0.741 & $\underline{0.725}$ \\
\hline Calidad $\mathrm{Q}^{2}$ & BI & 0.766 & 0.766 & 0.766 & $\overline{0.766}$ \\
\hline Calidad $\mathrm{Q}^{2}$ & PEOU & 0.694 & $\underline{0.690}$ & $\underline{0.690}$ & $\underline{0.690}$ \\
\hline Calidad $\mathrm{Q}^{2}$ & PU & 0.782 & 0.783 & 0.782 & 0.782 \\
\hline Calidad $\mathrm{Q}^{2}$ & S & 0.788 & 0.797 & 0.797 & \\
\hline Calidad $\mathrm{Q}^{2}$ & SU & 0.620 & 0.630 & 0.630 & $\underline{0.616}$ \\
\hline
\end{tabular}

Tabla 4. Comparativo de índices de calidad del modelo. Nota: Índice subrayado indica desmejora

\section{Discusión y conclusión}

La facilidad con la que los estudiantes usan la tecnología hoy en día, se evidencia por la influencia positiva de la auto-eficacia computacional (CSE) en la facilidad de uso percibida (PEOU), según muestra este estudio. Esto coincide con los resultados que se obtuvieron en otras investigaciones similares (Al-Mushasha \& Ieee, 2013; Arteaga \& Duarte, 2010; Cheng, 2011; Hsiao et al., 2013; Kiliç, 2014; Y.-H. Lee, Hsieh \& Ma, 2011; Mbarek \& Zaddem, 2013; Ong, Lai \& Wang, 2004; RamirezAnormaliza et al., 2015).

La utilidad percibida (PU) se destaca como el factor externo más importante que influye en el comportamiento de la intención de utilizar los sistemas e-learning en los estudiantes de grado ecuatorianos, en tanto que se calcula un coeficiente de ruta de 0.62 , siendo el segundo la facilidad de uso percibida (PEOU), para el cual se calcula un 0.34, observándose que ambos constructos tienen significancia estadística (ver Figura 3).

En este estudio la influencia social (SI) resulta tener un efecto directo sobre la utilidad percibida (PU). Este resultado es similar a lo que obtuvieron Abbad et al. (2009), Udzlmd et al. (2014) y Cheng (2011) 
en sus estudios. Este hallazgo da la pauta que para tener una mejor aceptación de los sistemas e-learning entre los estudiantes de grado en Ecuador, es importante conseguir que los líderes o referentes en la organización usen primero éstas herramientas, impulsando así su utilización entre el resto de los miembros de la organización.

El presente estudio evidencia que las relaciones fundamentales (hipótesis) del TAM se confirman en Ecuador, al igual de lo que se ha observado en otros países. Esto induce a pensar que en términos generales se observa en el Ecuador pautas parecidas a las observadas a nivel internacional, si bien con algún matiz relacionado con el contexto ecuatoriano.

Así por ejemplo, el soporte técnico (TS) no tiene influencia sobre la utilidad percibida (PU) y la facilidad de uso percibida (PEOU) en la aceptación y uso de los sistemas e-learning, al contrario de lo observado en otros estudios de este tipo (Abbad et al., 2009; AlQudah Ahmed, 2014; Arteaga \& Duarte, 2010), lo cual puede deberse a que cada vez los estudiantes se encuentran más capacitados en el uso de ordenadores. Esto coincide con lo que se discutió anteriormente al identificar la influencia positiva de CSE sobre PU y PEOU, en tanto que,al encontrase los estudiantes mejor capacitados en el uso de las TIC es razonable pensar que no requieran de soporte técnico (o requieran poco).

Un segundo matiz a destacar es la influencia positiva de Utilidad Percibida (PU) sobre la Satisfacción (S), dado el alto valor del coeficiente de ruta (0.93) observado. Esta relación fue específicamente validada en el apartado anterior y tiene el interés de ser un resultado que difiere de lo que suele observarse en estudios anteriores, que muestran coeficientes de ruta menores. Se trataría pues de una aportación bastante novedosa de este estudio (Calli et al., 2013; T.-C. Lin \& Chen, 2012).

El resultado anterior indicaría que las expectativas del estudiante en que el sistema e-learning mejore la adquisición de conocimiento y competencias en el proceso de enseñanza-aprendizaje (constructo Utilidad Percibida - PU) se traducen en una alta satisfacción ante la constatación de que el sistema e-learning es eficiente y tiene las características necesarias para alcanzar tales objetivos (constructo Satisfacción - S). El resultado obtenido implica un reconocimiento por parte de los estudiantes de que la introducción de estos sistemas ha impactado positivamente en el proceso de enseñanza-aprendizaje. Ante la posibilidad de que este resultado se deba a un cierto solapamiento entre los constructos PU y se procedió a revisar qué ítems podrían ser responsables de ello. Se identificó a S2 y S3 (Ver Anexo 1) como posibles fuentes de solape y tras eliminarlos se procedió a re-evaluar el modelo empíricamente. El resultado final mantiene un coeficiente de ruta superior a 0.9 , lo cual da validez al modelo inicial. 
También se identificó, para el caso de Ecuador, la influencia positiva del entretenimiento percibido (PE) sobre la facilidad de uso percibida (PEOU), de forma similar a lo observado por Abbad et al. (2009), Udzlmd et al. (2014) y Cheng (2011).Las causas de este resultado podrían explicarse por la edad de los encuestados, en tanto que, al tratarse de usuarios jóvenes puedan valorar de forma especial el disfrute con el uso de la tecnología. Este fenómeno justifica plantear nuevos estudios que aporten un modelo más innovador y adaptado a un perfil de usuario tipo nativo digital, en los que se incorporen constructos como "computer playfulness" (que podría traducirse como espontaneidad en el uso de ordenador) en el modelo (Al-Gahtani, 2016; Sahouly \& Rashid, 2015). Este sería un planteamiento más innovador respecto a la utilización del modelo TAM.

Siguiendo con las limitaciones del estudio, cabe comentar que la encuesta fue aplicada a estudiantes de una sola universidad estatal, lo cual disminuye las alternativas de análisis, puesto que en el Ecuador existen distintas categorías de universidad con características diversas. Así mismo, esto puede haber dejado de lado la posibilidad de analizar el comportamiento de estudiantes de las diferentes regiones de Ecuador, así como las opiniones de estudiantes de universidades privadas, que indudablemente pertenecen a estratos económicos diferentes y tienen características culturales diferentes. La constatación de estas limitaciones supone la necesidad de realizar nuevos estudios a nivel nacional, en los que se puedan realizar análisis comparativos por género, nivel de estudios (grado y posgrado), categorías de universidad, condiciones socio-económicas de los estudiantes, tipos de universidad (públicas y privadas) y diferencias de edad; así como estudios adicionales centrados en estudiar el efecto que pueda tener ciertas variables de control. De hecho, se está ya realizando un estudio a nivel nacional con las características mencionadas, en base al modelo utilizado en este estudio.

El hecho de haber considerado sólo la perspectiva de los estudiantes es también una limitación del presente estudio, en tanto que el éxito de un sistema e-learning no depende sólo de su aceptación y uso por parte de los estudiantes, sino que también debe tenerse en cuenta a los profesores. Resultan necesarios pues, nuevos estudios que incorporen el factor profesores. El estudio a nivel nacional que se comentaba anteriormente, también incorpora la perspectiva de los profesores.

Concluir que el estudio es un aporte a un mejor entendimiento de los factores que puedan potenciar el uso de los sistemas e-learning y en el caso concreto de los estudios de grado en Ecuador. Los resultados facilitarán a las directivos de las universidades tomar mejores decisiones en relación a la gestión de estos sistemas, en pro de incrementar los beneficios éstos puedan aportar. Este es el principal aporte de la presente investigación, en tanto no se evidencian trabajos similares en el contexto ecuatoriano. 
Así por ejemplo, los directivos deben tener en cuenta que para evaluar la aceptación de los sistemas e-learning entre los estudiantes universitarios de grado en Ecuador, además de los constructos básicos del TAM, la influencia social, el disfrute percibido,la auto-eficacia computacional y la satisfacción se constatan como factores clave. Por otra parte, los resultados obtenidos evidencian que las universidades no sólo deben preocuparse por el diseño básico de los sistemas e-learning (aspectos tecnológicos), sino que también deben abordar explícitamente las diferencias individuales entre los estudiantes universitarios que utilizan dichas herramientas, para así maximizar los beneficios que éstas ofrecen.

\section{Referencias}

Abbad, M.M., Morris, D., \& de Nahlik, C. (2009). Looking under the Bonnet: Factors Affecting Student Adoption of e-learning Systems in Jordan. International Review of Research in Open and Distance Learning, 10(2), 25. https://doi.org/10.19173/irrodl.v10i2.596

Adam, M., Vallés, R., \& Rodríguez, G. (2013). E-learning: Características y evaluación. Ensayos de Economía, 43, 143-159. Disponible online en: http://upcommons.upc.edu/bitstream/handle/2117/27314/42932-198754-1-PB.pdf?sequence=1

Al-Gahtani, S.S. (2016). Empirical investigation of e-learning acceptance and assimilation: A structural equation model. Applied Computing and Informatics, 12(1), 27-50. https://doi.org/10.1016/j.aci.2014.09.001

Al-Mushasha, N.F., \& Ieee. (2013). Determinants of e-Learning Acceptance in Higher Education Environment Based on Extended Technology Acceptance Model. 2013 Fourth International Conference on e-Learning Best Practices in Management, Design and Development of E-Courses: Standards of Excellence and Creativity, 261-266. https://doi.org/10.1109/econf.2013.50

AlQudah Ahmed, A. (2014). Accepting moodle by academic staff at the university of Jordan: Applying and extending tam in technical support factors. European Scientific Journal, 10(18), 183-200.

Antón, C., Camarero, C., \& San José, R. (2013). Public Employee Acceptance of New Technological Processes. Public Management Review, (June 2014), 1-24. https://doi.org/10.1080/14719037.2012.758308

Arteaga, R., \& Duarte, A. (2010). Motivational factors that influence the acceptance of Moodle using TAM. Computers in Human Behavior, 26(6), 1632-1640. https://doi.org/10.1016/j.chb.2010.06.011

Bagozzi, R.P. (2007). The Legacy of the Technology Acceptance Model and a Proposal for a Paradigm Shift. Journal of the Association for Information Systems, 8(4), 244-254. 
Bandura, A. (1977). Self-efficacy: Toward a unifying theory of behavioural change. Psychological Review, 84(2), 191-215. https://doi.org/10.1037/0033-295X.84.2.191

Brown, I., \& Inouye, D. (1978). Learned helplessness through modeling. Journal of Personality and Social Psychology, 36(8), 900-908. Disponible online en: http://psycnet.apa.org/index.cfm? fa=buy.optionToBuy\&id=1980-01003-001 https:// doi.org/10.1037/0022-3514.36.8.900

Calli, L., Balcikanli, C., Calli, F., Cebeci, I., \& Seymen, F. (2013). Identifying Factors that Contribute to the Satisfaction of Students in e-Learning. Turkish Online Journal of Distance Education, 14(1), 3-11.

Caporarello, L., \& Sarchioni, G. (2014). E-learning: The recipe for success. Journal of E-Learning and Knowledge Society, 10(1), 117-128.

Carmines, E.G., \& Zeller, R.A. (1994). Reliability and validity assessment. In J. L. Sllivan \& R. G. Niemi (Eds.), Quantitative Applications in the Social Sciences (Vol. 17). Berverly Hill - USA: McCune, Sara. https://doi.org/10.1037/018269

Chen, J.L. (2011). The effects of education compatibility and technological expectancy on e-learning acceptance. Computers and Education, 57(2), 1501-1511. https://doi.org/10.1016/j.compedu.2011.02.009

Cheng, Y.M. (2011). Antecedents and consequences of e-learning acceptance. Information Systems Journal, 21(3), 269-299. https://doi.org/10.1111/j.1365-2575.2010.00356.x

Chih-Yang, C., Tsai-Chu, C., Ping-Teng, L., \& Chih-Wei, L. (2011). A study of the Civil Servants' continuance of using e-learning. In 2011 International Conference on Information Technology Based Higher Education and Training, ITHET 2011. IEEE. https://doi.org/10.1109/ithet.2011.6018682

Cho, V., Cheng, T.C.E., \& Hung, H. (2009). Continued usage of technology versus situational factors: An empirical analysis. Journal of Engineering and Technology Management, 26(4), 264284. https://doi.org/10.1016/j.jengtecman.2009.10.003

Chou, J.-S., \& Tseng, H.-C. (2011). Establishing expert system for prediction based on the projectoriented data warehouse. Expert Systems with Applications, 38(1), 640-651. https://doi.org/10.1016/j.eswa.2010.07.015

Cohen, J. (1988). Statistical Power Analysis for the Behavioral Sciences (Second). Lawrence Erlbaum.

CONEA. (2009). Evaluación de Desempeño Institucional de las Universidades y Escuelas Politécnicas del Ecuador. Quito - Ecuador. 
Davis, F.D. (1985). A technology acceptance model for empirically testing new end-user information systems: Theory and results. Massachusetts Institute of Technology. Massachusetts Institute of Technology. Disponible online en: http://dspace.mit.edu/handle/1721.1/15192

Davis, F.D. (1989). Perceived Usefulness, Perceived Ease of Use, and User Acceptance of lnformation Technology. MIS Quarterly, 13(3), 319-340. https://doi.org/10.2307/249008

Davis, F.D., Bagozzi, R.P., \& Warshaw, P.R. (1992). Extrinsic and Intrinsic Motivation to Use Computers in the Workplace1. Journal of Applied Social Psychology, 22(14), 1111-1132. https://doi.org/10.1111/j.1559-1816.1992.tb00945.x

Davis, F.D., Bagozzi, R.P., \& Warshaw, R.P. (1989). User Acceptance of Computer Technology: A Comparison of Two Theoretical Models. Management Science, 35(8), 982-1003.

Dishaw, M.T., \& Strong, D.M. (1999). Extending the technology acceptance model with tasktechnology fit constructs. Information and Management, 36(1), 9-21. https://doi.org/10.1016/S03787206(98)00101-3

Duenas-Rugnon, O.L., Iglesias-Pradas, S., \& Hernandez-Garcia, A. (2010). System and User Characteristics in the Adoption and Use of e-Learning Management Systems: A Cross-Age Study. In M.D. Lytras, P.O. DePablos, D. Avison, J. Sipior, Q. Jin, W. Leal, ... D. Horner (Eds.), Technology Enhanced Learning: Quality of Teaching and Educational Reform (Vol. 73, pp. 301-307). Book Section, Berlin: Springer-Verlag Berlin. https://doi.org/10.1007/978-3-642-13166-0_43

Elkaseh, A.M., Wong, K.W., \& Fung, C.C. (2015). The Acceptance of E-learning as a Tool for Teaching and Learning in Libyan Higher Education. International Journal of Information Technology, 3(4), 1-11.

Feneche, T. (1998). Using perceived ease of use and perceived usefulness to predict accptance of the world wide web. Computer Networks and ISDN Systems, 30, 629-630. https://doi.org/10.1016/S01697552(98)00028-2

Findik, D., \& Kunçay, C.O.Ş. (2009). A model for instructors adoption of learning management systems : Empirical validation in higher education context. Middle East Technical University.

Fishbein, M., \& Ajzen, I. (1975). Belief, attitude, intention and behavior: An introduction to theory and research. Reading - Massachusetts: Addison-Wesley, Ed.

Fornell, C., \& Larcker, D.F. (1981). Evaluating Structural Equation Models with Unobservable Variables and Measurement Error. Journal of Marketing Research, 18(1), 39-50. https://doi.org/10.2307/3151312 
Fu, J.-R., Farn, C.-K., \& Chao, W.-P. (2006). Acceptance of electronic tax filing: A study of taxpayer intentions. Information \& Management, 43(1), 109-126. https://doi.org/10.1016/j.im.2005.04.001

Garson, G.D. (2016). Partial Least Squares: Regression \& Structural Equation Models (2016th ed.). Asheboro - USA: Statistical Associates Publishing.

Gómez Cruz, M.E. (2011). Estimación de los modelos de ecuaciones Estructurales, del indice mexicano de la satisfacción del usuario de programas sociales mexicanos, con la metodología de minimos cuadrados parciales. Univeresidad Iberoamericana.

Henseler, J., Hubona, G., \& Ray, P.A. (2016). Using PLS Path Modeling in New Technology Research: Updated Guidelines. Industrial Management \& Data Systems, 116(1), 2-20. https://doi.org/10.1108/IMDS09-2015-0382

Hsiao, J.-L., Wu, W.-C., \& Chen, R.-F. (2013). Factors of accepting pain management decision support systems by nurse anesthetists. BMC Medical Informatics and Decision Making, 13(1), 16. https://doi.org/10.1186/1472-6947-13-16

Hulland, J. (1999). Use of partial least squares (PLS) in strategic management research: A review of four recent studies. Strategic Management Journal, 20(2), 195-204. https://doi.org/10.1002/(SICI)10970266(199902)20:2<195::AID-SMJ13>3.0.CO;2-7

Humaidi, N. (2013). Exploratory Factor Analysis of User's Compliance Behaviour towards Health Information System's Security. Journal of Health \& Medical Informatics, 4, 2-9. https://doi.org/10.4172/2157-7420.1000123

Jeffrey, D.A. (2015). Testing the Technology Acceptance Model 3 ( TAM 3 ) with the Inclusion of Change Fatigue and Overload, in the Context of Faculty from Seventh-day Adventist Universities: A Revised Model. Andrews University.

Kiliç, E. (2014). Determining the Factors of Affecting the Moodle Use by Using TAM . The Story of a University after a Destructive Earthquake. Journal of Education, 29(October 2011), 169-179.

Kocaleva, M. (2015). Model of e-Learning Acceptance and Use for Teaching Staff in Higher Education Institutions. I.J. Modern Education and Computer Science, 4(April), 23-31. https://doi.org/10.5815/ijmecs.2015.04.03

Kripanont, N. (2006). Using a Technology Acceptance Model to Investigate Academic Acceptance of the Internet. Journal of Business Systems, Governance and Ethics, 1(2), 13-28. 
Landry, B.J. L., Griffeth, R., \& Hartman, S. (2006). Measuring Student Perceptions of Blackboard Using the Technology Acceptance Model. Decision Sciences Journal of Innovative Education, 4(1), 87-99. http://doi.org/10.1111/j.1540-4609.2006.00103.x

Lay, J.-G., Chen, Y.-W., \& Chi, Y.-L. (2013). GIS Adoption Among Senior High School Geography Teachers in Taiwan. Journal of Geography, 112(3), 120-130. https://doi.org/10.1080/00221341.2012.682226

Lee, J.-K., \& Lee, W.-K. (2008). The relationship of e-Learner's self-regulatory efficacy and perception of e-Learning environmental quality. Computers in Human Behavior, 24(1), 32-47. https://doi.org/10.1016/j.chb.2006.12.001

Lee, J.W. (2010). Online support service quality, online learning acceptance, and student satisfaction. Internet and Higher Education, 13(4), 277-283. https://doi.org/10.1016/j.iheduc.2010.08.002

Lee, M.-C. (2010). Explaining and predicting users' continuance intention toward e-learning: An extension of the expectation-confirmation model. Computers \& Education, 54(2), 506-516. https://doi.org/10.1016/j.compedu.2009.09.002

Lee, Y.-H., Hsieh, Y.-C., \& Ma, C.-Y. (2011). A model of organizational employees' e-learning systems acceptance. Knowledge-Based Systems, 24(3), 355-366. https://doi.org/10.1016/j.knosys.2010.09.005

Lee, Y. H., Hsieh, Y. C., \& Hsu, C.N. (2011). Adding Innovation Diffusion Theory to the Technology Acceptance Model: Supporting Employees' Intentions to use E-Learning Systems. Educational Technology \& Society, 14(4), 124-137.

Legris, P., Ingham, J., \& Collerette, P. (2003). Why do people use information technology? A critical review of the technology acceptance model. Information \& Management, 40, 191-204. https://doi.org/10.1016/S0378-7206(01)00143-4

Li, N., \& Kirkup, G. (2007). Gender and cultural differences in Internet use: A study of China and the UK. Computers \& Education, 48(2), 301-317. https://doi.org/10.1016/j.compedu.2005.01.007

Liao, H.L., \& Lu, H.P. (2008). Richness versus parsimony antecedents of technology adoption model for e-learning websites. In F. Li, J. Zhao, T. K. Shih, R. Lau, Q. Li, \& D. McLeod (Eds.), Advances in Web Based Learning - Icwl 2008, Proceedings (Vol. 5145, pp. 8-17). Book Section, Berlin: Springer-Verlag Berlin. https://doi.org/10.1007/978-3-540-85033-5_2

Liaw, S.-S., Chang, W.-C., Hung, W.-H., \& Huang, H.-M. (2006). Attitudes toward search engines as a learning assisted tool: Approach of Liaw and Huang's research model. Computers in Human Behavior, 22(2), 177-190. https://doi.org/10.1016/j.chb.2004.09.003 
Liaw, S.-S., Huang, H.-M., \& Chen, G.-D. (2007). Surveying instructor and learner attitudes toward elearning. Computers \& Education, 49(4), 1066-1080. Disponible online en: http://dl.acm.org/citation.cfm? id=1285049 https: / / doi.org/10.1016/j.compedu.2006.01.001

Lin, T.-C., \& Chen, C.-J. (2012). Validating the Satisfaction and Continuance Intention of e-learning Systems. International Journal of Distance Education Technologies, 10(1), 44-54.

https://doi.org/10.4018/jdet.2012010103

Lin, W.S. (2012). Perceived fit and satisfaction on web learning performance: IS continuance intention and task-technology fit perspectives. International Journal of Human-Computer Studies, 70(7), 498-507. https://doi.org/10.1016/j.ijhcs.2012.01.006

Little, B.B. (2009). Quality Assurance for Online Nursing Courses. Journal of Nursing Education, 48(7), 381-387. https://doi.org/10.3928/01484834-20090615-05

Lobato Rubio, V. (2008, November 20). Caracterización del conocimiento en PYMES que realizan proyectos de TIC: Un modelo de análisis y valoración. Tesis, Oviedo: Universidad de Oviedo. Disponible online en: http://dialnet.unirioja.es/servlet/tesis?codigo=19576\&info=resumen

Ma, Q., \& Liu, L. (2004). The technology acceptance model: A meta-analysis of empirical findings. Journal of Organizational and End User Computing, 16(1), 59-72. https://doi.org/10.4018/joeuc.2004010104

Mallak, L.A. (2001). Challenges in Implementing e-Learning. In International Conference on Management of Engineering and Technology (pp. 298-299). Portlan - Usa. https://doi.org/10.1109/picmet.2001.952186

Mbarek, R., \& Zaddem, F. (2013). The examination of factors affecting e-learning effectiveness. International Journal of Innovation and Applied Studies, 2(4), 423-435. http://doi.org/2028-9324

Mcgill, T., Klobas, J., \& Renzi, S. (2011). LMS Use and Instructor Performance: The Role of Tasktechnology Fit. International Journal on e-Learning, 10(1), 43-62.

Melas, C.D., Zampetakis, L.A., Dimopoulou, A., \& Moustakis, V. (2011). Modeling the acceptance of clinical information systems among hospital medical staff: an extended TAM model. Journal of Biomedical Informatics, 44(4), 553-564. https://doi.org/10.1016/j.jbi.2011.01.009

Mirriahi, N., Unit, T., Vaid, B.S., \& Burns, D.P. (2015). Winter / hiver 2015 Meeting the challenge of providing flexible learning opportunities: Considerations for technology adoption amongst academic staff Relever le défi de fournir des occasions d'apprentissage flexibles: Considérations pour l' adoption. Canadian Journal of Learning and Technology, 41(1), 1-16.

Ngai, E.W.T., Poon, J.K.L., \& Chan, Y.H.C. (2007). Empirical examination of the adoption of WebCT using TAM. Computers \& Education, 48(2), 250-267. https://doi.org/10.1016/j.compedu.2004.11.007 
Ong, C.-S., Lai, J.-Y., \& Wang, Y.-S. (2004). Factors affecting engineers' acceptance of asynchronous elearning systems in high-tech companies. Information \& Management, 41(6), 795-804. https://doi.org/10.1016/j.im.2003.08.012

Padilla-Meléndez, A., Águila-obra, A.R., \& Garrido-Moreno, A. (2015). Empleo de moodle en los procesos de enseñanza-aprendizaje de dirección de empresas: Nuevo perfil del estudiante en el eees. Educación XX1, 18(1), 125-146. http://doi.org/10.5944/educXX1.18.1.12314

Park, N., Lee, K.M., \& Cheong, P.H. (2007). University instructors' acceptance of electronic courseware: An application of the technology acceptance model. Journal of Computer-Mediated Communication, 13(1), 163-186. https://doi.org/10.1111/j.1083-6101.2007.00391.x

Parker, A. (1999). A Study of Variables that Predict Dropout from Distance Education. International Journal of Educational Technology, 1, 1-12. http://doi.org/Proquest:62307270

Peral Peral, B., Arenas Gaitán, J., \& Ramón-Jerónimo, M.Á. (2014). Technology Acceptance Model y mayores: ¿La educación y la actividad laboral desarrollada son variables moderadoras?. Revista Española de Investigación En Marketing ESIC, 18(1), 43-56. https://doi.org/10.1016/S1138-1442(14)60005-X

Peterson, R. (2000). A Meta-Analysis of Variance Accounted for and Factor Loadings in Exploratory Factor Analysis. Marketing Letters, 11(3), 261-275. Disponible online en: http://link.springer.com/article/10.1023/A\%3A1008191211004 https://doi.org/10.1023/A:1008191211004

Ramirez-Anormaliza, R., Sabaté, F., \& Guevara-Viejo, F. (2015). Evaluating student acceptance level of elearning systems. In ICERI2015 (Ed.), 8th annual International Conference of Education, Research and Innovation (pp. 2393-2399). Sevilla - Spain: IATED. Disponible online en; http://library.iated.org/view/RAMIREZANORMALIZA2015EVA

Ramirez-Correa, P.E., Javier Rondan-Cataluña, F., Arenas-Gaitán, J., \& Alfaro-Perez, J.L. (2016). Moderating effect of learning styles on a learning management system's success. Telematics and Informatics, 34, 272-286. https://doi.org/10.1016/j.tele.2016.04.006

Rashotte, L. (2007). Social influence. The Blackwell Encyclopedia of Social Psychology, (1985), 4.

Rey Martín, C. (2000). La satisfacción del usuario: Un concepto en alza. Anales de Documentación, 3, 139-153.

Sahin, I., \& Shelley, M. (2008). Considering Students ' Perceptions: The Distance Education Student Satisfaction Model. Educational Technology \& Society, 11(3), 216-223.

http://doi.org/10.2307/jeductechsoci.11.3.216 
Sahouly, I. Al, \& Rashid, T. (2015). Resurrecting Information Technology Adoption Models: A Theoretic Foundation. International Journal of Research in Management \& Technology (IJRMT), 5(6), 400-408.

Sanjuán Suárez, P., Pérez García, A.M., \& Bermúdez Moreno, J. (2000). Escala de autoeficacia general: Datos psicométricos de la adaptación para población española. Psicothema, 12(SUPPL. 2), 509-513. http://doi.org/ISSN 0214-9915

Schumacker, R.E., \& Lomax, R.G. (2010). A Beginner's Guide to Structural Equation Modeling (Taylor \& Francis Gropu, Ed.) (3rd ed.). New York - USA: Routledge. Disponible online en: http://books.google.com/books?id=58pWPxWPC90C\&pgis=1

Šumak, B., Heričko, M., \& Polančič, G. (2011). A meta-analysis of e-learning technology acceptance: The role of user types and e-learning technology types. Computers in Human Behavior, 27(6), 2067-2077. https://doi.org/10.1016/j.chb.2011.08.005

Šumak, B., Heričko, M., Pušnik, M., \& Polančič, G. (2011). Factors affecting acceptance and use of moodle: An empirical study based on TAM. Informatica, 35(1), 91-100.

Tarhini, A., Hone, K., \& Liu, X. (2013). Factors Affecting Students ' Acceptance of e-Learning Environments in Developing Countries: A Structural Equation Modeling Approach. International Journal of Information and Education Technology, 3(1), 54-59. https://doi.org/10.7763/IJIET.2013.V3.233

Tawfik, M., Sancristobal, E., Martin, S., Gil, R., Pesquera, A., Garcia, F. et al. (2013). Upcoming Challenges in e-Learning \& Online Learning Environments. In Society for Information Technology \& Teacher Education International Conference (Vol. 2013, pp. 1060-1065). Association for the Advancement of Computing in Education (AACE). Disponible online en: http://www.editlib.org/p/48259/

Tsai, C.C., Chuang, S.C., Liang, J.C., \& Tsai, M.J. (2011). Self-efficacy in internet-based learning environments: A literature review. Educational Technology and Society, 14(4), 222-240.

Udzlmd, D., Bachtiar, F.A., Rachmadi, A., \& Pradana, F. (2014). Acceptance in the Deployment of Blended Learning as Learning Resource in Information Technology and Computer Science Program, Brawijaya University. In Asia-Pacific Conference on Computer Aided System Engineering (APCASE) (pp. 131-135). IEEE.

University of Oxford International Strategy Office (2015). International Trends in Higher Education. University of Oxford, 25.

Venkatesh, V. (2000). Determinants of Perceived Ease of Use: Integrating Control, Intrinsic Motivation, and Emotion into the Technology Acceptance Model. Information Systems Research, 11(4), 342-365. https://doi.org/10.1287/isre.11.4.342.11872 
Venkatesh, V., \& Bala, H. (2008). Technology Acceptance Model 3 and a Research Agenda on Interventions. Decision Sciences, 39(2), 273-315. https://doi.org/10.1111/j.1540-5915.2008.00192.x

Venkatesh, V., \& Davis, F.D. (1996). A Model of the Antecedents of Perceived Ease of Use: Development and Test. Decision Sciences, 27(3), 451-481. https://doi.org/10.1111/j.1540-5915.1996.tb01822.x

Venkatesh, V., Morris, M., Davis, G., \& Davis, F. (2003). User acceptance of information technology: Toward a unified view. MIS Quarterly, 27(3), 425-478.

Welch, A.G., Ray, C.M., \& Peterson, C.M. (2015). The Role of Peer Influence and Perceived Teaching Quality in Faculty Acceptance of Web-Based Learning Management Systems. International Journal on e-Learning, 14(4), 487-524.

Weng, C., \& Tsai, C. (2015). Social support as a neglected e-learning motivator affecting trainee 's decisions of continuous intentions of usage. Australasian Journal of Educational Technology, 31(2), 177-192. https://doi.org/10.14742/ajet.1311

Williams, M.D., Nripendra, R.P., \& Dwived, Y.K. (2015). The unified theory of acceptance and use of technology (UTAUT): A literature review. Journal of Enterprise Information Management, 28(3), 443-488. https://doi.org/10.1108/JEIM-09-2014-0088

Wood, R., \& Bandura, A. (1989). Social Cognitive Theory of Organizational Management.. Academy of Management Review, 14(3), 361-384. https://doi.org/10.5465/AMR.1989.4279067

Yi, M.Y., \& Hwang, Y. (2003). Predicting the use of web-based information systems: Self-efficacy, enjoyment, learning goal orientation, and the technology acceptance model. International Journal of Human-Computer Studies, 59(4), 431-449. https://doi.org/10.1016/S1071-5819(03)00114-9

\section{Anexos}

\section{Anexo 1. Constructos - ítems}

\begin{tabular}{|c|c|c|}
\hline \multicolumn{2}{|c|}{ Constructos - ítems } & Fuente \\
\hline \multicolumn{3}{|c|}{ Soporte técnico (TS) } \\
\hline TS1 & El sistema e-learning proporciona ayuda cuando hay un problema técnico. & \multirow{6}{*}{$\begin{array}{c}\text { (Arteaga \& } \\
\text { Duarte } \\
\text { 2010) }\end{array}$} \\
\hline TS2 & $\begin{array}{l}\text { Una línea telefónica está disponible en cualquier momento para atender problemas } \\
\text { técnicos con el sistema e-learning. }\end{array}$ & \\
\hline TS3 & $\begin{array}{l}\text { Se pueden hacer preguntas por correo electrónico cuando se presenta un problema } \\
\text { técnico con el sistema e-learning. }\end{array}$ & \\
\hline TS4 & El sistema e-learning ofrece respuestas a preguntas frecuentes sobre su uso. & \\
\hline TS5 & $\begin{array}{l}\text { Se pueden hacer consultas en línea tipo chat con personas de soporte técnico } \\
\text { cuando hay algún problema con el sistema e-learning. }\end{array}$ & \\
\hline TS6 & $\begin{array}{l}\text { El personal de soporte del sistema e-learning tiene una predisposición positiva o } \\
\text { actitud de ayuda cuando es consultado. }\end{array}$ & \\
\hline
\end{tabular}




\begin{tabular}{|c|c|c|}
\hline \multicolumn{2}{|c|}{ Constructos - ítems } & Fuente \\
\hline \multicolumn{3}{|c|}{ La auto-eficacia computacional (CSE) } \\
\hline CSE1 & $\begin{array}{l}\text { Puedo completar las tareas de aprendizaje en el sistema e-learning si no hay nadie } \\
\text { alrededor para decirme qué hacer a medida que avanzo. }\end{array}$ & \multirow{9}{*}{$\begin{array}{c}\text { (Mbarek \& } \\
\text { Zaddem, } \\
\text { 2013), }\end{array}$} \\
\hline CSE2 & $\begin{array}{l}\text { Puedo completar las tareas de aprendizaje en el sistema e-learning si yo nunca } \\
\text { había usado un sistema informático como este antes. }\end{array}$ & \\
\hline CSE3 & $\begin{array}{l}\text { Puedo completar las tareas de aprendizaje en el sistema e-learning si tuviera sólo } \\
\text { los manuales de referencia del sistema. }\end{array}$ & \\
\hline CSE4 & $\begin{array}{l}\text { Puedo completar las tareas de aprendizaje en el sistema e-learning si he visto a } \\
\text { alguien más usar el sistema antes que yo. }\end{array}$ & \\
\hline CSE5 & $\begin{array}{l}\text { Puedo completar las tareas de aprendizaje en el sistema e-learning si alguien más } \\
\text { me ayuda con una inducción en el sistema. }\end{array}$ & \\
\hline CSE6 & $\begin{array}{l}\text { Una formación adicional en manejo de e-learning mejoría mi auto-eficacia } \\
\text { computacional y con ello el manejo del sistema e-learning. }\end{array}$ & \\
\hline CSE7 & $\begin{array}{l}\text { Gracias a mis cualidades y recursos puedo superar situaciones imprevistas dentro } \\
\text { del manejo del sistema e-learning. }\end{array}$ & \\
\hline CSE8 & $\begin{array}{l}\text { Puedo resolver la mayoría de los problemas que se presentan en el uso del sistema } \\
\text { e-learning si me esfuerzo lo necesario. }\end{array}$ & \\
\hline CSE9 & $\begin{array}{l}\text { Si me encuentro en una situación difícil en el manejo del sistema e-learning, } \\
\text { generalmente se me ocurre que debo hacer. }\end{array}$ & \\
\hline \multicolumn{3}{|c|}{ Influencia social (SI) } \\
\hline SI1 & $\begin{array}{l}\text { Mis profesores piensan que debería participar en las actividades de aprendizaje } \\
\text { basadas en el sistema e-learning que ellos realizan. }\end{array}$ & \multirow{5}{*}{$\begin{array}{c}\text { (Tarhini, } \\
\text { Hone \& } \\
\text { Liu, 2013) }\end{array}$} \\
\hline SI2 & $\begin{array}{l}\text { Mis compañeros de clase piensan que yo deberá participar en las actividades de } \\
\text { aprendizaje basadas en el sistema e-learning. }\end{array}$ & \\
\hline SI3 & $\begin{array}{l}\text { Los directivos de mi universidad consideran que debo realizar actividades de } \\
\text { aprendizaje basadas en el sistema e-learning. }\end{array}$ & \\
\hline SI4 & En términos generales, me gustaría hacer lo que mi profesor cree que debo hacer. & \\
\hline SI5 & $\begin{array}{l}\text { Las personas cuyas opiniones valoro (amistades, familia, etc.), piensan que debo } \\
\text { utilizar el sistema e-learning. }\end{array}$ & \\
\hline \multicolumn{3}{|c|}{ Entretenimiento Percibido (PE) } \\
\hline PE1 & $\begin{array}{l}\text { El sistema e-learning ha conseguido que mi estrés por conocer la calificación baje, } \\
\text { al conocerla inmediatamente. }\end{array}$ & \multirow{5}{*}{$\begin{array}{l}\text { (Weng \& } \\
\text { Tsai, 2015) }\end{array}$} \\
\hline PE2 & Pienso que usando el sistema e-learning la calificación es más imparcial. & \\
\hline PE3 & Me agrada más la nota calificada por el sistema e-learning, que por el profesor. & \\
\hline PE4 & Encuentro el sistema e-learning entretenido. & \\
\hline PE5 & Me gusta usar el sistema e-learning. & \\
\hline \multicolumn{3}{|c|}{ Influencia social (SI) } \\
\hline SI1 & $\begin{array}{l}\text { Mis profesores piensan que debería participar en las actividades de aprendizaje } \\
\text { basadas en el sistema e-learning que ellos realizan. }\end{array}$ & \\
\hline $\mathrm{SI} 2$ & $\begin{array}{l}\text { Mis compañeros de clase piensan que yo deberá participar en las actividades de } \\
\text { aprendizaje basadas en el sistema e-learning. }\end{array}$ & \\
\hline SI3 & $\begin{array}{l}\text { Los directivos de mi universidad consideran que debo realizar actividades de } \\
\text { aprendizaje basadas en el sistema e-learning. }\end{array}$ & $\begin{array}{l}\text { (1)arnini et } \\
\text { al., 2013) }\end{array}$ \\
\hline SI4 & En términos generales, me gustaría hacer lo que mi profesor cree que debo hacer. & \\
\hline SI5 & $\begin{array}{l}\text { Las personas cuyas opiniones valoro (amistades, familia, etc.), piensan que debo } \\
\text { utilizar el sistema e-learning. }\end{array}$ & \\
\hline Entreteni & ento Percibido (PE) & \\
\hline PE1 & $\begin{array}{l}\text { El sistema e-learning ha conseguido que mi estrés por conocer la calificación baje, } \\
\text { al conocerla inmediatamente. }\end{array}$ & \\
\hline PE2 & Pienso que usando el sistema e-learning la calificación es más imparcial. & (Weng \& \\
\hline PE3 & Me agrada más la nota calificada por el sistema e-learning, que por el profesor. & Tsai, 2015) \\
\hline PE4 & Encuentro el sistema e-learning entretenido. & \\
\hline PE5 & Me gusta usar el sistema e-learning. & \\
\hline
\end{tabular}




\begin{tabular}{|c|c|c|}
\hline \multicolumn{2}{|c|}{ Constructos - ítems } & Fuente \\
\hline \multicolumn{3}{|c|}{ Satisfacción (S) } \\
\hline S1 & $\begin{array}{l}\text { Estoy satisfecho de utilizar el sistema e-learning como herramienta para de } \\
\text { aprendizaje. }\end{array}$ & \multirow{6}{*}{$\begin{array}{l}\text { (W.S. Lin, } \\
\text { 2012) }\end{array}$} \\
\hline S2 & El sistema e-learning es eficiente para la construcción del conocimiento. & \\
\hline S3 & El sistema e-learning es eficiente para el intercambio de conocimiento. & \\
\hline S4 & Estoy satisfecho con la interactividad de los cursos que utilizanelsistema e-learning. & \\
\hline S5 & $\begin{array}{l}\text { Estoy satisfecho con las diversas opciones de evaluación que ofrece el sistema e- } \\
\text { learning. }\end{array}$ & \\
\hline S6 & $\begin{array}{l}\text { Me satisface el uso de los sistemas e-learning, porquecontribuye al desarrollo de las } \\
\text { competencias transversales, como el trabajo en equipo. }\end{array}$ & \\
\hline \multicolumn{3}{|c|}{ Utilidad Percibida (PU) } \\
\hline PU1 & En los cursos en que se utiliza el sistema e-learning, mejora mi rendimiento. & \multirow{5}{*}{$\begin{array}{l}\text { (Lay, Chen, } \\
\text { \& Chi, } \\
\text { 2013) }\end{array}$} \\
\hline PU2 & En los cursos en que se utiliza el sistema e-learning, el aprendizaje es eficaz. & \\
\hline PU3 & $\begin{array}{l}\text { En los cursos en que se utiliza el sistema e-learning, se hace más fácil el } \\
\text { aprendizaje. }\end{array}$ & \\
\hline PU4 & $\begin{array}{l}\text { En los cursos en que se utiliza el sistema e-learning, el sistema es de utilidadpara el } \\
\text { aprendizaje. }\end{array}$ & \\
\hline PU5 & Es conveniente en el aprendizaje, el uso del sistema e-learning. & \\
\hline \multicolumn{3}{|c|}{ Facilidad de Uso Percibido (PEOU) } \\
\hline PEOU1 & Aprender a operar el sistema e-learning resulta muy fácil. & \multirow{5}{*}{$\begin{array}{c}\text { (Lay et al., } \\
\text { 2013) }\end{array}$} \\
\hline PEOU2 & La interacción con el sistema e-learning es clara y comprensible. & \\
\hline PEOU3 & Encuentro que el sistema e-learning es flexible para interactuar con él. & \\
\hline PEOU4 & Sería fácil ser competente en el uso del sistema e-learning. & \\
\hline PEOU5 & Encuentro que el sistema e-learning es fácil de usar. & \\
\hline \multicolumn{3}{|c|}{ Intención hacia el uso (BI) } \\
\hline BI1 & $\begin{array}{l}\text { En cuanto sea posible utilizar el sistema e-learning en mis cursos que tengan como } \\
\text { soporte este sistema. }\end{array}$ & \multirow{5}{*}{$\begin{array}{c}\text { (Findik \& } \\
\text { Kunçay, } \\
2009 \text { ) }\end{array}$} \\
\hline BI2 & Es importante usar el sistema e-learning, recomendaría su utilización. & \\
\hline $\mathrm{BI} 3$ & En cuanto sea posible usaré el sistema e-learning el próximo semestre & \\
\hline BI4 & $\begin{array}{l}\text { Estoy impaciente para entregar las tareas en el sistema e-learning antes del plazo } \\
\text { establecido. }\end{array}$ & \\
\hline $\mathrm{BI} 5$ & Me gustaría utilizar el sistema e-learning en el futuro si tuviera la oportunidad. & \\
\hline \multicolumn{3}{|c|}{ Uso del Sistema (SU) } \\
\hline SU1 & $\begin{array}{l}\text { En término de horas semanales, tiendo a utilizar el sistema e-learning un tiempo } \\
\text { razonable a lo que propone el modelo docente de mi asignatura. }\end{array}$ & \multirow{6}{*}{$\begin{array}{c}\text { (Peral Peral, } \\
\text { Arenas } \\
\text { Gaitán, \& } \\
\text { Ramón- } \\
\text { Jerónimo, } \\
\text { 2014) }\end{array}$} \\
\hline SU2 & Me paso tiempo explorando dentro del sistema e learning. & \\
\hline SU3 & Me involucro con el sistema de e-learning. & \\
\hline SU4 & $\begin{array}{l}\text { Ingreso al sistema e-learning para interactuar en mis cursos, al menos una vez al } \\
\text { día. }\end{array}$ & \\
\hline SU5 & $\begin{array}{l}\text { Me conecto con frecuencia al sistema e-learning para comprobar si hay novedades } \\
\text { relativas a mis asignaturas }\end{array}$ & \\
\hline SU6 & $\begin{array}{l}\text { Me conecto con frecuencia al sistema e-learning para avanzar en mis tareas } \\
\text { asignadas dentro del sistema. }\end{array}$ & \\
\hline
\end{tabular}




\section{Anexo 2. Resumen de las escalas de medición}

\begin{tabular}{|c|c|c|c|c|c|}
\hline Constructosítems & Media & $\begin{array}{l}\text { Desviación } \\
\text { Estándar }\end{array}$ & $\begin{array}{c}\text { Carga } \\
\text { factorial }\end{array}$ & $\begin{array}{l}\text { Fiabilidad } \\
\text { compuesta }\end{array}$ & $\begin{array}{c}\text { Varianza } \\
\text { media extraída }\end{array}$ \\
\hline \multicolumn{4}{|l|}{ Soporte Técnico (TS) } & 0.96 & 0.80 \\
\hline TS1 & 4.98 & 1.74 & 0.86 & & \\
\hline TS2 & 4.41 & 2.02 & 0.87 & & \\
\hline TS3 & 4.73 & 1.94 & 0.89 & & \\
\hline TS4 & 4.76 & 1.89 & 0.94 & & \\
\hline TS5 & 4.68 & 1.95 & 0.90 & & \\
\hline TS6 & 4.97 & 1.81 & 0.89 & & \\
\hline \multicolumn{4}{|c|}{ Auto-eficacia Computacional(CSE) } & 0.97 & 0.81 \\
\hline CSE1 & 5.41 & 1.57 & 0.91 & & \\
\hline CSE2 & 5.29 & 1.61 & 0.87 & & \\
\hline CSE3 & 5.34 & 1.55 & 0.91 & & \\
\hline CSE4 & 5.44 & 1.55 & 0.91 & & \\
\hline CSE5 & 5.53 & 1.52 & 0.88 & & \\
\hline CSE6 & 5.55 & 1.53 & 0.90 & & \\
\hline CSE7 & 5.54 & 1.49 & 0.91 & & \\
\hline CSE8 & 5.38 & 1.55 & 0.91 & & \\
\hline CSE9 & 5.28 & 1.58 & 0.88 & & \\
\hline \multicolumn{4}{|l|}{ Influencia Social (SI) } & 0.95 & 0.80 \\
\hline SI1 & 5.27 & 1.65 & 0.91 & & \\
\hline $\mathrm{SI} 2$ & 5.02 & 1.70 & 0.91 & & \\
\hline SI3 & 5.26 & 1.64 & 0.89 & & \\
\hline SI4 & 5.13 & 1.63 & 0.89 & & \\
\hline SI5 & 5.19 & 1.63 & 0.88 & & \\
\hline \multicolumn{4}{|c|}{ Entretenimiento Percibido (PE) } & 0.96 & 0.81 \\
\hline PE1 & 5.30 & 1.68 & 0.86 & & \\
\hline PE2 & 5.26 & 1.63 & 0.89 & & \\
\hline PE3 & 5.07 & 1.72 & 0.91 & & \\
\hline PE4 & 5.07 & 1.74 & 0.92 & & \\
\hline PE5 & 5.15 & 1.70 & 0.92 & & \\
\hline \multicolumn{4}{|l|}{ Satisfacción (S) } & 0.98 & 0.91 \\
\hline S1 & 5.29 & 1.67 & 0.94 & & \\
\hline S2 & 5.34 & 1.61 & 0.96 & & \\
\hline S3 & 5.37 & 1.55 & 0.95 & & \\
\hline S4 & 5.29 & 1.58 & 0.97 & & \\
\hline S5 & 5.33 & 1.55 & 0.96 & & \\
\hline S6 & 5.35 & 1.57 & 0.95 & & \\
\hline \multicolumn{4}{|c|}{ Utilidad Percibida (PU) } & 0.98 & 0.91 \\
\hline PU1 & 5.25 & 1.61 & 0.95 & & \\
\hline PU2 & 5.28 & 1.60 & 0.97 & & \\
\hline PU3 & 5.30 & 1.58 & 0.96 & & \\
\hline PU4 & 5.37 & 1.51 & 0.96 & & \\
\hline PU5 & 5.39 & 1.50 & 0.94 & & \\
\hline \multicolumn{4}{|c|}{ Facilidad de Uso Percibido (PEOU) } & 0.98 & 0.92 \\
\hline PEOU1 & 5.45 & 1.52 & 0.96 & & \\
\hline PEOU2 & 5.43 & 1.52 & 0.96 & & \\
\hline PEOU3 & 5.44 & 1.52 & 0.96 & & \\
\hline PEOU4 & 5.48 & 1.52 & 0.96 & & \\
\hline PEOU5 & 5.50 & 1.54 & 0.96 & & \\
\hline \multicolumn{4}{|c|}{ Intención hacia el uso (BI) } & 0.98 & 0.89 \\
\hline BI1 & 5.43 & 1.53 & 0.95 & & \\
\hline $\mathrm{BI} 2$ & 5.38 & 1.55 & 0.96 & & \\
\hline $\mathrm{BI} 3$ & 5.39 & 1.57 & 0.96 & & \\
\hline BI4 & 5.39 & 1.59 & 0.90 & & \\
\hline BI5 & 5.47 & 1.63 & 0.94 & & \\
\hline
\end{tabular}




\begin{tabular}{|c|c|c|c|c|c|}
\hline \multicolumn{4}{|c|}{ Uso del Sistema (SU) } & \multirow[t]{2}{*}{0.97} & \multirow[t]{2}{*}{0.84} \\
\hline SU1 & 5.30 & 1.64 & 0.93 & & \\
\hline SU2 & 4.99 & 1.68 & 0.90 & & \\
\hline SU3 & 5.13 & 1.66 & 0.94 & & \\
\hline SU4 & 5.14 & 1.75 & 0.90 & & \\
\hline SU5 & 5.37 & 1.67 & 0.93 & & \\
\hline SU6 & 5.32 & 1.70 & 0.91 & & \\
\hline
\end{tabular}

Intangible Capital, 2017 (www.intangiblecapital.org)

Article's contents are provided on an Attribution-Non Commercial 3.0 Creative commons license. Readers are allowed to copy, distribute and communicate article's contents, provided the author's and Intangible Capital's names are included. It must not be used for commercial purposes. To see the complete license contents, please visit http://creativecommons.org/licenses/by-nc/3.0/. 